\title{
The Stem Cells and the Cancer Stem Cells
}

\author{
Giuseppe Loverro $^{1}$, Gianluca Raffaello Damiani ${ }^{2 *}$, Matteo Loverro ${ }^{1}$, Maria Teresa Loverro ${ }^{1}$ and Erica Silvestris ${ }^{1}$ \\ ${ }^{1}$ Department of Obstetrics and Gynecology, University of Bari Aldo Moro, Italy \\ ${ }^{2}$ Department of Biomedical Sciences and Human Oncology, University of Bari Aldo Moro, Italy \\ Submission: April 15, 2019 ; Published: April 29, 2019 \\ *Corresponding author: Gianluca Raffaello Damiani, Department of Obstetrics and Gynecology, University of Bari Aldo Moro, Italy
}

Abbrevations: CAR: Receptor of Chimeric Antigen; OCSC: Stem Cells of Ovarian Cancer; NK: Natural Killer; iFN: Interferon; I: Interleukin; PBMC:

Peripheral Blood Mononuclear Cells

\section{Introduction}

The origin and the properties of Stem Cells (SCs) represent a large chapter of modern medicine that has been fascinated clinicians and biologists for about thirty years, principally in relation to the many evolutionary and innovative aspects related to these cells, which remain today a topic still unresolved. Two major characteristics define a SC as unique multiplastic cell, namely the self-renew capacity and the property to differentiate to multiple cell lines under specific signals. In fact, these cells, in suitable conditions and in contrast with other somatic cells undergoing a fixed number of divisions, show unlimited ability to divide into udefined number of copies mantaining the same characteristics [1]. Based on their capacity for self-renewal and differentiation, CSs can be classified into: Embryonic Stem Cells (ESC) and Adult Stem Cells (ASC).

\section{Embryonic stem cells (ESC)}

Embryonic stem cells are pluripotent cells that are potentially immortal. They derive from the inner cell mass (ICM) of the blastocysts and are characterized by the ability to self-renewal and the capability to generate all cells within the human body. Since the first isolation of ESCs huge interest has developed in the scientific and clinical communities for their therapeutic potential. In fact, particular attention has focused their potential use in cell-based therapy for diseases refractory to conventional treatments, such as neurodegenerative diseases and immunodeficiency, because of their ability to be programmed into new mature differentiated cells of different lineages [2].

\section{Adult stem cells (ASC)}

The recent identification of adult stem/progenitor cells in most tissues/organs in mammalian organisms, which provide critical functions in homeostatic maintenance by replenishing the mature cell types within the tissues in which they reside over the lifetime, has caused great interest and enthusiasm for their use in cellular and tissue engineering therapies. The ASCs are clonogenic, self-renewing, totipotent or multipotent cells with differentiating plasticity in cytotypes of the original tissue and often in others to transdifferentiate. Unlike the ESCs, which are defined by their origin from the ICM of the blastocyst, the origin of the ASCs is still under investigation.

\section{Pluripotent stem cells (iPSCs)}

The inducible pluripotent stem cells (iPSCs) are a third type of SCs, generated by the genic reprogrammation of somatic cells into a pluripotent state. Cellule staminali pluripotenti inducibili (iPSCs). The translation to clinical applications of these cells is a promising and rapidly emerging branch of regenerative medicine in which SC based treatments could be applied to treat and cure aggressive and lethal diseases in humans. Nevertheless, the therapeutic potential of adult stem cells as powerful tools in tissue regeneration and engineering has been recognized, and intense efforts are ongoing to harness and direct adult stem cell plasticity.

Although the stemness studies have greatly improved the basic knowledge of HSCs and MSCs in humans providing substantial progress in different cytotherapies as BM transplantation or anticancer approaches with engineered MSCs, poor information is presently available on the biology of germline stem cells (GSCs) whose existence is today still disputed although experimentally proven [3].

In the last two decades, great attention has also been adressed to the neoplastic counterpart of CSs or cancer stem cells (CSCs), which have become the object of scientific research, gaining increasing interest and preserving therapeutic hopes mainly in relation to their potential for target therapy in a tumor. The discovery of CSCs has undoubtedly led to a change in nosology and the conceptual approach to cancer. If up until now, a tumor has been conceived as a set of malignant cells with random renewal and 
differentiation properties, today two pathogenetic mechanisms that would be at the origin of a neoplasm are acceptable, the first based on CSCs and the second on clonal evolution.

The hypothesis that contemplates the CSCs admits a hierarchy among the tumor cells in which the CSCs at the apex would produce the tumor mass [4]. The second model of clonal evolution recognizes tumor heterogeneity as a result of genetic and epigenetic modifications that occurred during neoplastic progression. However, in relation to the data that are available today, the two models mentioned above cannot be considered exclusive, since the CSCs could also evolve over time, generating different clonal subpopulations within the tumor [5].

The origin of CSCs remains highly debated and controversial. Based on the functional and phenotypic similarities between them and the CSs (self-renewal, differentiation, quiescence, expression of markers, etc.), it was initially assumed that these cellular elements were a derivation of normal somatic SCs undergoing malignant transformation. Instead, more recent evidence, considers as an additional mechanism to the origin of CSCs, a dedifferentiation and reprogramming of the adult neoplastic cellular component even if this hypothesis needs further studies [6]. The CSCs are quiescent cells, arrested in the G0 phase of the cell cycle even though they have a high migratory capacity and therefore have the property of spreading away from the primary tumor to give metastasis, following the mesenchymal epithelial transition (MET) [7]. Furthermore, their organization in small clusters shows a certain resistance to chemo / radiotherapy with high metastatic capacity [8].

Although CSCs represent only a small tissue cell subpopulation, they constitute a cellular reserve capable of self-sustaining, selfrenewing and differentiating into heterogeneous cancer cells [9]. Therefore, in relation to these properties and to their partecipation in the genesis and recurrence of atumor, these cells are commonly called "cancer initiating stem cells". In this review we will try to outline the gene profile from the molecular point of view of the CSCs, following a detailed revisiting of the literature.

\section{OSCs}

The GSCs are a cellular population addressed to the production of gametes aimed at the propagation of the species. The concept of GSCs was introduced by Regaud, who postulated that for the occurence of spermatogenesis, a cell population with self-renewal properties could be present in the testicle able to produce differentiated progeny. It is known that these cells, now known as spermatogonic stem cells, contribute to adult spermatogenesis in males of all species studied. The search for the existence of a female counterpart, able to undergo postnatal neo-oogenesis and thus contribute to the production of oocytes in adulthood, has opened a larger debate.

Although female GSCs (fGSC) seem to play a role in oogenesis throughout reproductive life in some non-mammalian species, the presence of fGSC in mammals has been much discussed since the prevailing theory, suggested in the 19th century by Waldeyer who postulated that mammals are born with a finite stock of mature oocytes that are exhausted with aging [10]. The hypothesis of a fixed ovarian reserve in mammals, was subsequently contested by Tilly and his collaborators [11], who affirmed the presence of mitotically active ovarian stem cells (OSCs) in both juvenile and adult murine ovaries able to guarantee availability of oocytes and follicles after birth.

The first attempt to isolate and cultivate OSCs was completed by Zou and his collaborators who purified neonatal and adult OSCs from mice through a cell selection approach [12]. First, they performed a two-stage enzymatic digestion of the murine ovarian tissue followed by immunomagnetic isolation of the cells expressing the MVH germinal marker, also known as Ddx4 (polypeptide 4 of the DEAD box). This protein is a peptide of $79.3 \mathrm{kDa}$ with the extracellular domain of $\mathrm{COOH}$ and a cytoplasmic tale that is uniquely expressed by ovarian and spermatogonial germ cells [13]. After cultivating OSC from newborn and adult mice for more than 15 and 6 months respectively, the authors infected the Ddx-4+ cells isolated with the MSCV-PGK-GFP virus and transplanted them into ovaries of infertile female mice. Finally they observed that the animals produced GFP + / Ddx-4 + oocytes. Therefore, Tilly and his collaborators validated a FACS protocol to purify OSCs from the murine and human ovarian cortex as [14]. The investigation was based on the immunological detection of a superficial variant of Ddx-4, commonly considered cytoplasmic, through a rabbit polyclonal antibody against the terminal $\mathrm{COOH}$-protein. Once purified, the cells showed a pattern of germline gene expression and were then stabilized in culture, labeled with GFP and injected into adult human ovarian cortical tissue. The fragments were subsequently subjected to xenograft in immunodeficient female mice and new follicles containing positive GFP oocytes were detected 1-2 weeks later. The greater skepticism of this procedure emerged with respect to the choice of using Ddx-4 as a cell surface marker used for the selection of OSCs in relation to its apparently cytoplasmic location rather than on the plasma membrane [15]. Subsequently, Zhang and his group generated a fluorescent mouse model to trace OSCs expressing Ddx-4 in vivo and in vitro and, in the absence of fluorescence in the ovaries, supposed that the OSCs did not enter into mitosis and did not contribute to the renewal oocyte [16]. Another group of researchers described the presence of very small embryonic stem cells (VSELs) and germinal OSCs within the superficial ovarian epithelium (OSE) of adult mammals [13].

In the context of the pathophysiology of OSCs, the two main aspects that are attracting the attention of more and more researchers are the possible origin of gametes from SCs and the pathogenetic and clinical role of this cell population in its cancerous variant (OCSCs). On the first topic a definitive conclusion has not yet been reached, although in our latest work we have provided further evidence of the existence of OSCs in the ovarian cortex of women of childbearing age and in menopause and the ability of these cells to differentiate in vitro, forming cells 
oocyte-like mature. Using single cells isolated on the basis of their size, we were able to demonstrate that, in culture, larger cells were capable of expressing markers of terminal differentiation of the oocyte line (growth differentiation factor (GDF-9), synaptonemal complex protein ( SYCP3)), and entered in meiosis.

We therefore concluded that it is possible to generate oocytelike cells in vitro also from OSCs of menopausal women [17]. The potential uses of OSCs are numerous, particularly in basic sciences, but also in clinical applications, thanks to the main property of these cells to differentiate into mature and competent in vitro oocytes. It is admissible that OSCs can be used as a fertility preservation strategy in women who require gonadotoxic treatment for a cancer that could make them sterile, but also for the reversibility of reproductive senescence induced by infertility conditions related to age or ovarian failure premature (POI). No less promising are the knowledge obtained on the nature and on the influence that the neoplastic counterpart of the OSCs, or OCSCs would have in the origin and development of ovarian cancer.

The high rate of recurrence after treatments and the consequent still high lethality of the ovarian cancer, justify the great attention of many researchers that try to understand, more and more clearly, the nature and possible influences of OCSCs in the evolution of this tumor.

\section{OCSCs}

The CSCs are multipotent cells capable of self-renewing, mainly due to their property of SCs but also thanks to an intense activity of various cellular pathways capable of regulating their function, such as: Wnt, Hedgehog, Notch [18] and PI3K/Akt [19]. The isolation of OCSCs is a procedure that contains several difficulties, mainly in relation to the scarcity of the population and the absence of specific cellular markers.

The first successful experiment of isolation of OCSCs from the ascitic fluid of an ovarian neoplasm was published 2005 by Bapat et al. [20]. In the study, the authors report the develop of 19 clones of immortalized cells, which two transplanted into the SCID mouse (severe combined immunideficiency) were found to grow by developing tumors similar to the orginal cancer. Each single matured clone was then isolated and showing increased aggressiveness with respect to the parent tumor. This experiment was one of the first evidences capable of demonstrating the heterogeneous growth properties of SC subpopulations of cancer cells in ovarian tumor [20]. The OCSCs possess decisive characteristics in the evolution of ovarian cancer (OC), such as chemo-radio resistance relative to their quiescence status and to the expression of molecular pumps capable of expelling the drug, characteristics that give this tumor, not only migratory properties and invasive, but also resistance to therapy and high capacity for relapse [21].

Compared to this marked oncological aggressiveness, the percentage representation of OCSCs does not appear very high, as they represent only $0.9 \%$ of the total cell population [22].
The existence, the prognosis and the possible development of therapeutic strategies are correlated to the identification and expression of specific differentiation markers within the tumor stem component of every tumor, whose knowledge appears an important premise. In recent years, a large spectrum of these markers has been reported, which have been widely characterized by international literature, in order to better identify cancer stem cells and formulate a proper prognosis. However, it is necessary to underline that, unlike other tumors such as haematological ones, the diagnostic capacity to identify OCSCs through very precise differentiation markers is less reliable in ovarian tumors, due to their extreme heterogeneity.

\section{Identification of the Ocscs}

As previously described, the CSCs are characterized by surface markers specific to the tumor in which they reside. The most important markers suggestive of the oavrica nature of a CSCs are: CD44 + (hyaluronic acid receptor), CD24 +, CD117 + (c-kit), CD133 + (prominine) and ALDH1 + [23]. It is possible to identify and classify the CSCs by flow cytometry which therefore allows to obtain a phenotypic characterization of these cells [24]. In the ovarian caner, due to the strong variability, is not possible to delineate a single phenotype, therefore, a combined identification of several markers and the use of combined molecular characterization methods is preferable [25]. Therefore, further identification strategies could consist in the functional characterization of the "side-population" phenotypes (SP) (by expelling the vital dye Hoechst 33342 by the $\mathrm{ABC}$ transporters), in the changes of the enzymatic activities of the enzyme aldehyde dehydrogenase (ALDH) (by the method is a reagent called ALDEFLUOR, a substance which in the presence of high levels of this enzyme turns stem cells into fluorescence) and finally in the ability of OCSCs to grow in serum agar soils (soft agar medium), forming colonies similar to "Spheres" in suspension. Given the great variability of surface markers expressed by OCSCs, it is useful to analyze their nature and functions in order to develop a correct prognosis and an adequate therapeutic approach to treatment.

CD44: CD44 is a surface glycoprotein that acts as a receptor for hyaluronic acid, an important component of the extracellular matrix that contributes to cell proliferation, migration and progression in some malignant tumors. Once this glycoprotein has joined its primary ligand, hyaluronic acid (HA), it undertakes the adhesion of CSCs to the extracellular matrix, contributing to tumor migration and to the activation of a variety of transduction signals such as Nanog and EGFR-Ras-ERK [26]. The expression of CD44 is promoted during MET, which is essential for the acquisition of staminal characteristics of epithelial tumor cells [27]. The CSCs expressing CD44+ High and CD133+ High are highly radioresistant and possess a greater expression of other markers such as AKT (AKT1/2). Therefore, the CSCs-CD44+ High and CD133+ High compared to CD44 Low and CD133 Low have a marked ability to repair DNA and prevent apoptosis after radiotherapy, properties that make these cells a target for cytotoxic therapy [28]. 
CD 117 and c-Kit: CD 117 or c-Kit is co-expressed with CD44 and makes the cell capable of asymmetric division, correlating with chemoresistance.

CD 133 (prominin-1): CD133 is a membrane glycoprotein that gives rise to the formation of spheres and confers a high potential for differentiation. This surface marker is used to identify various types of cancer and in the ovarian cancer (OC) it can define the TIC (tumor initiating cell) population. The transfection of CD33+ cells, obtained from human OC, in non-obese diabetic/SCID mice, has shown that it is possible to generate a heterogeneous neoplasm similar to the original one [29]. The expression of CD133 is a factor of poor prognosis, whose expression correlates with a reduction in the survival rate in patients with CO [30].

ALDH1: The marker ALDH1 (aldehyde dehydrogenase1), if expressed, gives the CSCs the capacity for TIC, and if co-expressed with CD133 (CSCs CD133+/ALDH+) induces neoangiogenesis, with reduction of survival, which results proportionate to its intensity of expression [31]. The accumulation of CSCs-ALDH1+ after neoadjuvant chemotherapy would predict a resistance to treatment, associated with a worse prognostic than ovarian cancer [31].

CD4: CD4 can regulate the expression of Nanog and other stem cell-related genes, such as OCT4 with TIC properties. which leads to tumor initiation.

CD24: CD24 is considered a putative marker of OCSCs serous histotype, whose elevated expression would increase the quiescent, chemoresistance and neoplasm start properties. The CSCs-CD24+ express increased levels of typical CS genes such as Nestin, Beta-catenin, Bmi-1, Oct4, Oct3/4, Notch1, and Notch4 when compared with CD24-cells. Data literature show the association of CD24 with a reduction in microvascular density and cell proliferation, and with increased apoptosis [31].

\section{Targeting of OCSCs}

The Ovarian cancer has a tendency to relapse after a period of apparent disappearance, thus becoming a "tumor stem cell model" [32]. The identification and characterization of CSCs has made available multiple strategies also aimed at the treatment of ovarian cancer, mainly thanks to the advent of specific molecular therapies whose targets are defined as molecular pathways, functional components and environmental interactions of OCSCs [33]. The cancer targeted therapy uses the aid of drugs that differ from traditional chemotherapy, being directed against genes and/or proteins capable of stopping tumor progression [32]. Many strategies have been developed that can act against the OCSCs whose main ones are the following: inhibition of signaling pathways that play a key role in the activity of CSCs (inhibitors of signaling mechanisms such as Notch, Hedgeohog, Wnt)

a) gene silencing

b) targeting of surface markers using antibodies to CD133 and CD44

c) targeting of the tumor microenvironment (antivascularization, anti-hypoxia)

d) immunoevasion (antibodies and immune cells and cytokines)

e) targeting $\mathrm{ABC}$ cassettes (agents against $\mathrm{ABC}$ transporter and agents modulating the MDA, PgP, PCRP genes

f) epigenetic targeting

g) targeting by miRNAs

h) immunotherapeutic strategies

i) strategies based on nanoparticles (release of target agents using CART cells or combination therapy)

\section{Targeting of signaling pathways dependent on OCSCs}

One method to eliminate CSCs lies in the attack of "signaling pathways" including molecules specifically associated with control, self-renewal, cell differentiation and apoptosis (PI3K/ Akt, PTEN, JAK/STAT, Wnt/ $\beta$-catenin, hedgehog, Notch, and NFIoㅡ) Figure 1. Specifically, we will consider only those that are the object of ovarian targeting.

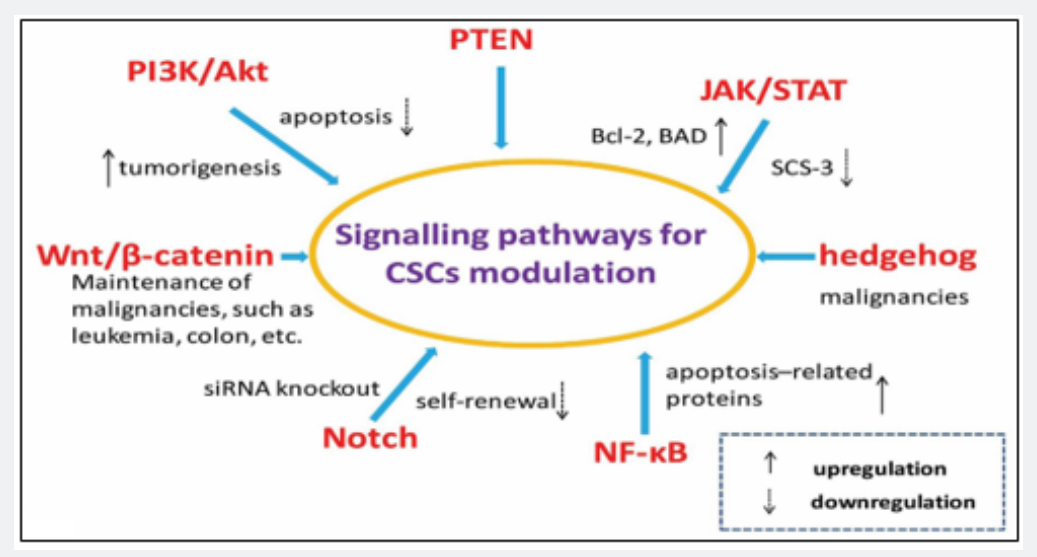

Figure 1: Molecular pathways related to CSCs. Common pathways to SCs and CSCs involved in the control of cell renewal and diffrenziation (PI3K/Akt, JAK/STAT, Wnt/B-catenin, hedgehog, Notch, NF-KB). 
Akt: The activation of the Akt signaling molecule plays a critical role for cell transformation and tumorigenesis. Literature data report an Akt correlation with the activation of the JAK/ STAT signaling pathway. Indeed, the study by Guo et al. shows that Akt1 increases apoptotic resistance by increasing the expression of Bcl-2 levels and phosphorylazone of the BAD pro-apoptotic protein, simultaneously activating the pathway JAK / STAT. In this context, the control of the suppressor of cytokine signaling-3 (SCS3 ) is seen as an effective method to regulate tumor formation by activating JAK / STAT [34].

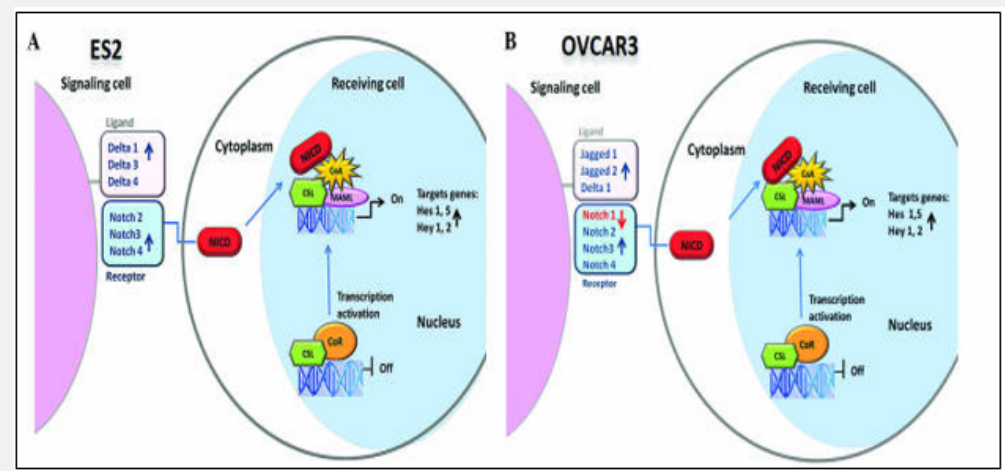

Figure 2: Description of the Notch pathway in the OC.

Notch: The Notch signaling pathways is interested in the development of OC, in the initiation of metastasization and in self-renewal processes. The over-expression of Notch3 can lead to greater expansion of OCSCs and to an emphasis on platinum chemoresistance in ovarian cancer [35]. The over-activation of the Notch pathway influences the MET, contributing to the invasiveness of the CSCs [36]. Notch3 is overexpressed in OCSC and was correlated with platinum resistance increase (Figure 2).

Hedgheog (Hh): In ovarian cancer the activity of the $\mathrm{Hh}$ pathway has been documented as a ptoential mechanism interested in the tumoral evolution [37]. Although a direct correlation between Hh expression and clinical stage is reported, it is still unclear whether the expression of Hh ligand is correlated with reduced survival [38]. The important role of Hh signaling in the cell proliferation of the $\mathrm{OC}$ is confirmed by the dose dependent reduction of the replicative potential after addition of monoclonal antibodies against Shh [39], suggesting that the activation of Hh could be a driver for $\mathrm{CO}$, making itself a target attractive for therapeutic inhibition.

Although still in an experimental state, it is known that the CO cell lines are susceptible to the inhibition of the Hh pathway as demonstrated by the strong arrest of their growth after treatment with cyclopamine [40]. The treatment with IPI-926, a cyclopamine derivative, inhibiting the growth of serous CO histotype, suggesting that Hh contributes to the pathogenesis of neoplasia and therefore represents a new therapeutic target especially in the maintenance setting.

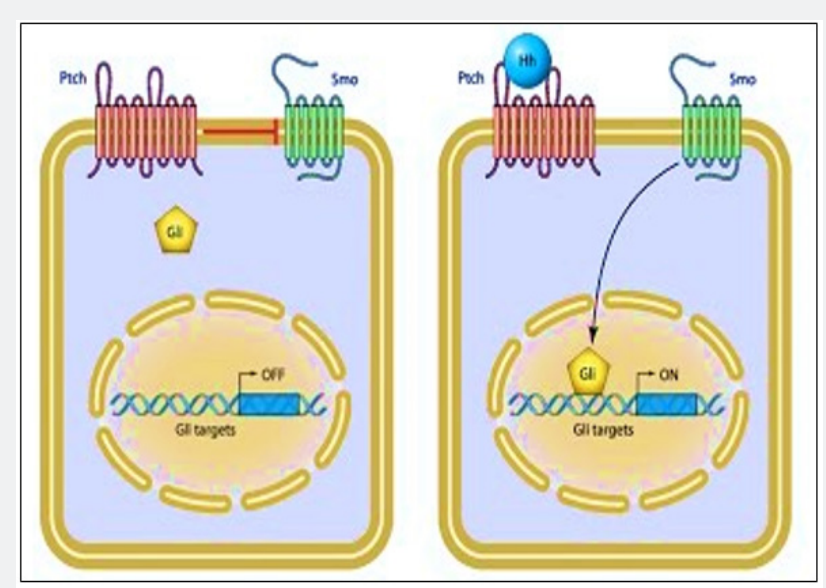

Figure 3: Pathway of $\mathrm{Hh}$ in the OC.

IPI-926 has demonstrated an antitumor activity both as a single agent and in maintenance therapy after administration of cytotoxic treatment in OC xenografts models. Although further studies are needed to determine precisely how the inhibition of Hh exerts this significant antineoplastic effect, the data currently presented indicate promising applications in the treatment of neoplasia. Finally, Hh anomalies are related to an aberrant activity of the Wnt pathway. In fact, we imagine that Wnt plays a role in maintaining CSCs' self-renewal [41] (Figure 3). 
Wnt: The therapies aimed at targeting and inhibiting the Wnt pathway are varied and interesting, even if still in an experimental way, as no drug has been approved for such use. The Wnt pathway would seem to have a link with the related Notch and Sonic Hedgehog pathways, with consequent important applications in the cancer treatment approach. Indeed, there are significant possibilities in Wnt targeting, including the detection of agents that do not interfere with the function of normal SCs, important in homeostasis and tissue repair. The Wnt-beta catenin pathway is extremely important for the maintenance of SCs and tissue and organ regeneration [42]. OMP-54 F28 (Ipafricept) is a drug currently tested in a phase Ib trial of ovarian carcinoma (NCT02092363), capable of inhibiting Wnt/b-catenin signaling targeting, having the Fzd8-Fc receptor as a molecular target.

\section{Gene silencing}

The RNA interference (RNAi) is a mechanism by which some RNA fragments are able to interfere (and shut down) gene expression, cause the degradation of complementary target mRNAs and thereby silencing the gene. Although there are numerous therapeutic strategies based on RNAi that affect the cells of the tumor mass, few studies have shown that they have the specific objective of CSCs RNAi. Despite this technique, following the silencing of selective genes, it has contained substantial promises as a new treatment modality in preclinical studies, its application in the field is also hampered by the inability to release sufficient doses into the tumor site. To this theory the Polymeric (the polymeric polymers are organic macromolecules that are characterized by a backbone chain of single bonds and dopppi) was considered as the most efficient vehicle for drug release, thanks to its pharmacokinetic properties. In the context of gene silencing, was developed the "targeted siRNAs", a technique that allows inducing a downregulation of the oncogenes of CSCs invivo. In breast cancer, the use of PEGylated EpCAM aptamens to guide siRNA to EpCAM overexpressing CSCs resulted in siRNA accumulation in the tumor site, resulting in an $80 \%$ annulment of the survivin gene (inhibitor of apoptosis and promoter of chemoresistance in the CSCs). Furthermore, the association of doxorubicin with the aptamero-siRNA chimera improved survival rates of tumor-affected mice, thus demonstrating the efficacy of anti-CSC RNAi in vivo as well as in vitro [43].

\section{Targeting of surface markers using antibodies for CD133, CD 44, Hedgehog, CD24, CD 117, CD326, ALDH}

Thanks to recent advances in the knowledge of CSCs biomarkers, the "target terapy" of tumors has had a new and growing impulse. The expression of specific CSC surface markers is heterogeneous and differs not only from patient to patient but also within the same tumor. It is therefore difficult not only to assess which markers are expressed by a single tumor at a specific stage of development, but also consequently the relative specific treatment to be applied. In any case, what is certain is that the surface markers of CSCs play important roles not only in the isolation and identification of the same, but also in the preclinical diagnosis of the neoplasm and in the treatment approach [44]. Ligands or antibodies against surface markers have been widely used to accentuate therapeutic strategies against cancer.

CD133: The development of drugs, aimed at eliminating CSC by targeting CD133 is still at a preliminary stage and clinical trials are still limited. It is known that the IP anti-CD133 toxin (dCD133KDEL) exerts a strong antitumor effect when it is injected intraperitoneally into the animal model of ovarian cancer (NIH: OVCAR5-luc), with consequent significant reduction in neoplastic progression [45]. Further preclinical studies show that anti-CD133 antibodies concentrate in ovarian tumors of xenotransplantation, thus suggesting that they can be used as agents or as drugs [46].

CD44: One of the biomarkers of the most used CSCs is CD44, that is a surface receptor of the extracellular matrix. Many studies have considered CD44 antibody therapy as the main anti-CSC approach. There are various monoclonal antibodies designated for the CD44 target in squamous cancers and adenocarcinomas [47]. In a mouse model, the CD44+ metastatic intraperitoneal cancer cells were treated with microparticles of a conjugated hyaluronic acid-paclitaxel (HA-TXL), with a significant reduction in weight and nodules [48]. Yang and his group in their work prove that the expression of CD44 from ovarian CSCs can be used to develop acid-coated nanoparticles capable of transporting Paclitaxel or a small interfering RNA (siRNA) to inhibit "multi drug resistance" genes ( MDR-1) [49].

Hedgehog: In the murine ovarian tumor xenograft model, the treatment of $\mathrm{CO}$ with Hedgehog antagonists is able to reduce tumor growth but above all inhibits tumor recurrence after treatment with carboplatin and paclitaxel [50].

CD24: CD24 plays an important role in metastases and tumor survival. The short hairpin RNA (small hairpin RNA) shRNA, is an RNA sequence that, when curved, forms a structure that resembles a hairpin and is usually used for silencing gene expression through the activation of RNA interference. It reduces the expression of CD24 with a consequent reduction in the growth of an ovarian tumor xenograft (Figure 4).

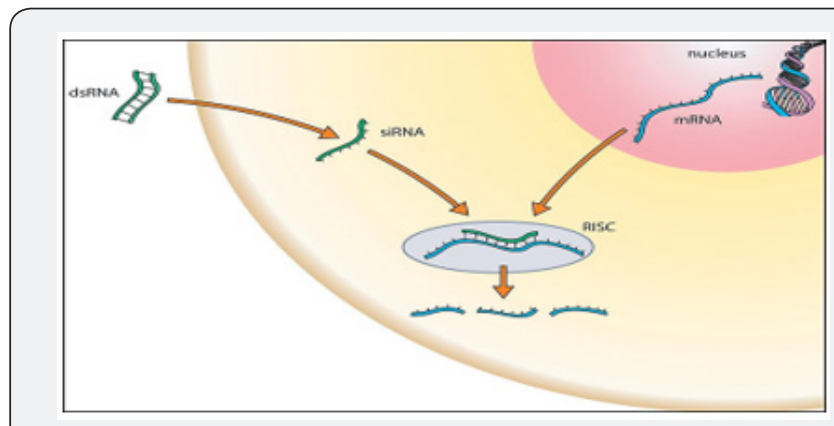

Figure 4: RNAi can be used in research: by administering siRNA from outside that is associated with RISC, it is possible to degrade specific mRNAs.

CD117: CD117 is an oncogene with tyrosine kinase receptor activity expressed on SCs, which represents an attractive target 
for cancer therapy. As tyrosine kinase, CD117 is a true 'drugable target' which is a protein that has a high affinity for drug binding.

Inhibitors of CD117 activity, such as imatinib, have been shown to be highly effective in gastrointestinal stromal tumors, but also against in vitro ovarian cancer cell lines [51]. Unfortunately, phase 2 clinical trials using imatinib in both relapsing disease and as a maintenance agent in patients after complete clinical remission showed no efficacy [52], and similarly, the combination of docetaxel and imatinib for the treatment of platinum ovarian cancer resistant showed few answers [53].

Noguera, in his study, evaluated imatinib mesylate, a specific CD117 inhibitor, in 13 patients with resistant low-grade platinum ovarian tumors in a phase II trial. $48 \%$ of these patients had positive c-kit utmore. Eleven patients were eligible for response assessment, and although the drug was well tolerated, no antitumor activity was observed in these low-grade tumors.

EpCam (CD 326): EpCam (CD326) is a $\mathrm{Ca}^{2+}$-independent cell adhesion molecule, expressed on the basolateral surface of most epithelial cells, which can characterize the CSCs of numerous neoplasms [54]. The trargeting of Epcam with the trifunctional Catumaxomab is approved for the intraperitoneal treatment of malignant ascites and is effective in the context of advanced ovarian cancers [55]. This anti-EpCAM monoclonal antibody was evaluated in a phase II/III trial in 258 patients with malignant epithelial cancer ascites, half of whom were ovarian carcinomas. When compared with paracentesis alone for the treatment of ascites, the addition of catumaxomab increased the time interval between two paracentesis (11dvs 77d, $P<0.0001$ ) [56]. Catumaxomab was also evaluated in combination with cytotoxic chemotherapy in a phase II trial (ENGOT-ov8) [57].

ALDH: The Disulfuran, an inhibitor of Aldehyde dehydrogenase (ALDH) has exerted controversial effects, acting with in vitro antineoplastic action but with little in vivo effects in murine models, probably due to its low bioavailability in vivo [58]. ALDHs are enzymes that promote the oxidation of aldehyde substrates to their corresponding $\alpha$-carboxylic acids. The ALDH family, composed of 19 isoforms with similar catalytic functions, includes the ALDH1 subgroup, particularly active in SCs and CSCs.

ALDH1 is considered a putative marker of OCSCs, whose upregulation correlates with an induction of chemoresistance [59] and whose downregulation could restore the chemosensitivity in ovarian cancer cell lines. Currently, many ALDH inhibitors are available. Among them, Disulfiram showed significant activity against CSCs in breast cancer and in others [60].

Since Disulfiram is an "FDA approved drug", it represents an important drug capable of testing the possible efficacy of the principle according to which ALDH inhibitors can exert a "targeting" action against CSCs in ovarian cancer. To all this it should be added that Disulfuram has been used safely in cancer patients, in combination with chemotherapy, deducing that SCs will tolerate therapies targeting ALDH [61]. To conclude, a marker capable of distinguishing OCSCs is never limited to ovarian cancer, but also extends to other types of cancer, therefore, to identify OCSCs, co-expression of "paired markers," such as Lgr5/ALDH1 is always required, CD44/CD117, and ALDH/CD133. The diversity of the couple reveals the sharing of various neoplasms and tumor heterogeneity [61].

\section{Targeting of the tumor microenvironment (anti- vascularization, anti-hypoxia)}

The tumor microenvironment (TM), which includes: surrounding blood vessels, cells of the immune system, connective cells and extracellular tumor matrix, is the cellular and extracellular environment around which the tumor exists and that of solid tumors is indicated by the term "tumor stroma". The tumor microenvironment contributes to tumor heterogeneity. One technique used in the discovery of new drugs is high-throughput screening, which uses in vitro cultured cancer cells. One of the weaknesses of the HTS techniques concerns the absence of experimentation on cells of the TM; only in recent times have new studies been developed that target cancer cells in vivo, thus also including the TM.

\section{Immunoevasion (antibodies and immune cells and cytokines)}

Despite its great potential, the immune system is often inable to eradicate a tumor, as the latter implements multiple immunoevasion mechanisms, which include the acquisition by cancer cells of the ability to resist immunological attack and/or alterations in the effector mechanisms of the immune system. Among the main mechanisms that directly affect tumor cells we can identify:

a) loss of expression of tumor antigens, especially the most immunogenic ones;

b) negative modulation of the molecules of the major histocompatibility complex (MHC) with consequent decrease of the antigenic presentation capacity [62]

c) insensitivity to IFN- $\gamma[63]$

d) defective signaling of death receptors, which contributes to tumor cell survival [64]

e) ability to induce lymphocyte death via the Fas death receptor pathway [65]

f) hyper-expression of inhibitory molecules of the cytotoxic activity of lymphocytes (eg PI-9 inactivates Granzima B; costimulatory molecule B7-H1 which can inhibit activated effector T cells [66]

g) ability of the tumor to modulate function of specific populations of the immune system, through factors or immunosuppressive cells.

In particular, the tumor could alter the function of DC by not providing adequate danger signals such as to induce its activation or could directly suppress its activity through the production of 
chemokines and cytokines (PG-E2, IL-10, TGF- $\beta$ ); the tumor also induces the development of suppressive myeloid cells (MDSC) capable of suppressing the proliferation of T CD4+ and CD8+ lymphocytes and of supporting neoplastic growth by promoting tumor neovascularization24. Finally, there are other populations of suppressive cells of the immune response, which can be recruited by the tumor (through the production of chemokines): regulatory $\mathrm{T}$ cells (Treg, CD4+ CD25+) that could prevent the generation and activation of $\mathrm{T}$ effector cells directed against cells autologous tumors, and the same NKT cells that, despite having an anti-tumor role, are also able to inhibit the activity of cytotoxic T lymphocytes (CTL) by producing IL-13 [67].

Finally the same Ag-specific $\mathrm{T}$ cells could be functionally unable to migrate to the tumor site and to infiltrate the anergic tumor, and not responsive or unable to completely differentiate in the tumor environment [68]. The expression of TAA antigens by human tumors and the awareness that the immune system has the potential to recognizing and eliminating tumor cells (immunosurveillance of the tumor) constitute the rationale for the development of immunotherapeutic strategies to mediate the regression of stabilized tumors.

The requirements for successful immunotherapy are basically three:

a) presence, in the host, of a sufficient number of tumorreactive lymphocytes, with high avidity

b) ability of lymphocytes to migrate to the tumor site and infiltrate the neoplastic mass

c) ability of lymphocytes to fully perform their effector function to eliminate tumor cells33. An "ideal" immunotherapy should also be specific (capable of discriminating between normal and tumor cells), sensitive (capable of eliminating even a single residual cancer cell), lasting (capable of providing protection against possible recurrence).

With these assumptions, various immunotherapeutic strategies have been tested to date on humans and the approaches used can be classified into two categories: active immunotherapy (which aims to induce an anti-tumor response in the host) and passive immunotherapy (which consists of in the transfer, in the host, of cellular or humoral effectors directed against tumor cells).

\section{Targeting $\mathrm{ABC}$ cassettes (agents against $\mathrm{ABC}$ transporter and agents modulating the MDA, PgP genes)}

The chemoresistance of CSCs is due in large part to the overexpression of drug efflux pumps such as the "Adenosine triphosphate-binding cassette (ABC) transporters", for which various pharmacological agents have shown inhibitory or neutralizing effects of these transporters. Three generations of inhibitors of the main $\mathrm{ABC}$ transporters are reported, of which the most specific one is P-glycoprotein (P-gp). To date, no one has been guaranteed for clinical use, due to the lack of specificity and numerous side effects. Recently, a more specific P-gp inhibitor, vardenafil, appeared promising in mitigating the side effects of P-gp overexpression, resulting in increased intracellular concentration and cytotoxicity of paclitaxel and vincristine [69].

\section{RNAi was also used to suppress ABC transporter genes}

The siRNA targeting P-gp has been shown to have reversed the famous resistance in breast cancer. Indeed, by exposing the siCNA-resistant CSCs to P-gp, a downregulation of the P-gp gene expression with doxorubicin intracellular accumulation and a resensitization equal to 4-fold was obtained [70]. Actually it is possible to affirm with certainty that nanotechnology can be used alone or in combination with drugs or RNAi directed towards ABC transporters.

It is known that CSCs possess an efflux system capable of deactivating the activity of chemotherapy drugs, resulting in the formation of a highly drug resistant tumor. The CSCs express several ABC-transporters like the ABCB1/P-gp/MDR1, ABCG2/ BCRP/MXR, and ABCB5, which are strongly dependent on the generation of ATP in the CSCs. Therefore, glycolysis targeting or depletion of ATP production in CSC should lead to inhibition of ABC transporters.

Since ABCG2 is considered as a high-speed vector of various substratesincluding chemotherapeutic agents, theCSC-ABCG2+are the most drug-resistant. It has been suggested that the expression of ABCG2 is upregulated by hypoxia through the transcription of the hypoxia-inducible transcription factor complex HIF- $1 \alpha$ and HIF- $2 \alpha$ signaling. Inhibition of ABC transporters may also cause toxicity to the patient's normal SCs, as they possess a pronounced DNA repair mechanism.

\section{Epigenetic targeting of CSCs}

Given that CSCs carry epigenetic alterations, it has been hypothesized that DNA hypomethylating agents can reprogram these cells to a more differentiated phenotype. Therefore, secondgeneration DNA methyltransferase inhibitors, especially SGI-110, were tested on ALDH+ ovarian CSCs, resulting in the restoration of sensitivity to platinum derivatives, up-regulation of genes associated with differentiation and increased tumor induction time in mouse [71].

\section{Targeting by miRNA}

Another possible therapeutic strategy is the use of miRNAs. MicroRNAs (miRNAs) are small endogenous single-stranded noncoding RNA molecules, that are active in the regulation of gene expression at the transcriptional and post-transcriptional level, which therefore play a central role in the initiation, progression, invasion and metastasis of cancer.

The miRNAs are incorporated into the RNA-induced silencing complex (RISC) and induce gene silencing by overlapping with complementary sequences present on target messenger RNA (mRNA) molecules. This link involves a repression of the translation or degradation of the target molecule Figure 5. 


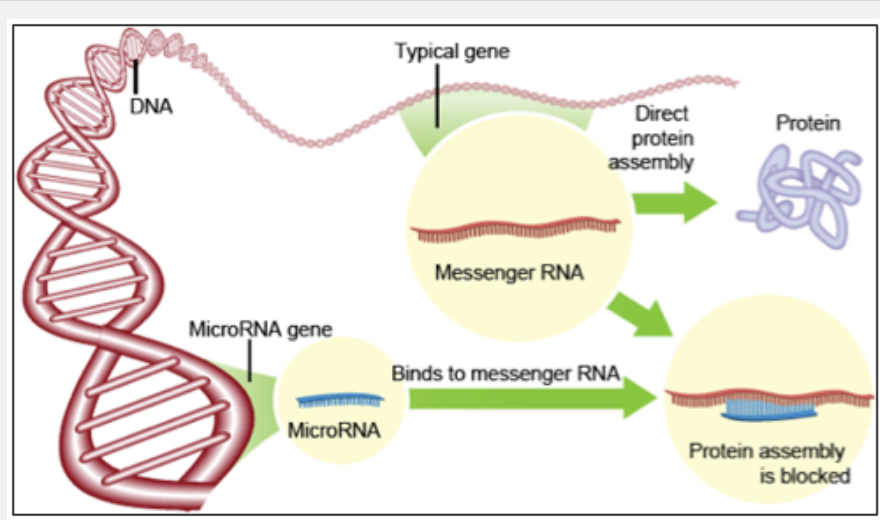

Figure 5: Graphic representation of a miRNA.

The miRNAs implied in the modulation of the transformation of CSCs and in the EMT are miR-200a, b, c; miR23b, miR 27a, b; miR 346.424, 503; miR214; miR199a; miR 125; miR138; miR 141; miR429; MiR 34; miR20a. The miRs implicated in neoangiogenesis are $\operatorname{miR} 145$; miR125b; MiR 484, 642, 217. Finally, miR355 increases extracellular matrix epithelium (ECM). Below we will describe the most important ones for the CO.

miR-200c: It is present in the SKOV3 OC CD44 + / CD117 + cell line subpopulation and is involved in the inhibition of EMT [72]. In fact, it determines a significant down regulation of the expression of ZEB-1 and vimentin, which leads to an up-regulation of E-cadherin and to a dramatic reduction in the capacity of colony formation, migration, and invasion in vitro, with reduction of the extent of metastasis in the CS11 CS117 + CD44 + xenograft models [72].

miR-214: It appears to be increased in OCSCs and interferes with the P53/Nanog axis, contributing to their confluence and self-renewal capacity [73].

miR-23b, miR-27a, miR-27b, miR-346, miR-424 e miR503: Expressed in CO-ALDH1+chemoresistant cells. The miR-27 expression levels correlate with the extent of CO metastasis [74].

miR-199a: In human OCs, this miRNA represses CD 44 expression and inhibits proliferation, migration, and invasion of CD44 + CD117 + ovarian CSCs. The inhibition of CD44 by miR199a reduces the expression of the ABCG2 gene by multidrug resistance, increasing the chemosensitivity of ovarian CSCs [75].

miRNA-125a: It is considered as a tumor suppressor in CO, inhibiting the MET process in the context of highly invasive OC cells [76].

miR-138: Down-regulated in invasive OC cell lines, in vivo its overexpression was able to inhibit OC metastases [77]. miR-138 directly represses the expression of genes associated with the EMT phenotype as SRY-related high-mobility group box (SOX4) and hypoxia-inducible factor- $1 \alpha$ (HIF- $1 \alpha)$.

miR-34: It is a Tumor Suppressor transactivated by P53 with reduced expression in OC cells compared to normal tissue, especially if disease progression is present [78]. Some miRs are involved in cell-ECM interactions and in the expression of ECM components and in angiogenesis.

miR-145: Repressed in OC cell lines due to its inhibitory effect on neo-angiogenesis [79] is capable of repressing the expression of p70S6K1 and therefore also that of HIF-1 $\alpha$ and VEGF.

In conclusion, it appears that miRs are capable of altering the state of metastases by modulating the expression of genes involved in the transformation of CSCs, MET, angiogenesis, and ECM, revealing promising markers for $\mathrm{OC}$, although further studies are needed to demonstrate its diagnostic and therapeutic role.

\section{Immunotherapeutic strategies}

The CSCs are capable of generating an immune evasion in order to grow, differentiate and generate both the primary and the metastatic lesion. There are many immunotherapeutic strategies for the trageting of CSCs in various types of cancers, such as NK cells, therapeutic vaccine, monoclonal antibodies (mAb), and the "blockade of immune checkpoints". Actually, all these immunotherapeutic strategies have proven to be effective in experimental ovarian cancer, although the road to their use in clinical practice is still long.

Allogeneic immunotherapy with NK cells: The NK cells can affect and eliminate CSCs in a manner not conditioned by the major histocompatibility complex (MHC). Cell immunotherapy was obtained in many cancers, including breast cancer [80]. One study reported that NK cells activated by IL- 2 and IL-15 are effective in eliminating the CDC-CD24 human breast cancer CSCs [81]. In a recent case report, NK cells were isolated from the patient's peripheral blood, and up to 8-10 billions were increased. The activated cells were expanded ex vivo for 14 days before intravenous infusion. After six infusions of NK cell therapy for more than 3 months, the level of Ca 125 decreased significantly (from 11.270 to $580 \mathrm{U} / \mathrm{ml}$ ). Furthermore, the size of the masses in the abdomen was severely reduced. Globally, this treatment had few adverse effects and prolonged the patient's survival [82].

Therapeutic vaccine for cancer: The therapeutic vaccine for cancer requires the participation of the innate and adaptive 
immunity of the patients through a first stimulation of the host with a tumor-specific antigen to activate the removal of the tumor [83]. The cancer vaccine is superior to other therapies in which the immunological memory exerts a relapse prevention for a certain period and may have implications in the field of therapy for OCSCs. $\mathrm{Wu}$ and his collaborators reported that the SKOV3 CD117+CD44 CSC cell line vaccine results in regression of ovarian cancer in xenotrapinated mice. This vaccine can therefore reduce the positive CSCs for CD117CD44ed and ALDH1 in immunized mice with IFN- $\gamma$ accentuation, reduction of TGF- level, and increase in the cytotoxic activity of NK cells [84].

Monoclonal antibodies (mAb): Monoclonal antibody immunotherapy $(\mathrm{mAb})$ uses an antigen-antibody response to develop the host's immunocompetence in order to remove target cells. The underlying mechanism is to activate cell-dependent antibody-cytotoxicity mediated and a complement-dependent cytotoxicity, inhibit the mediated receptor signaling, prepare antigen-presenting cells, effector cells and memory T cells [85]. Many preclinical and clinical analyzes are currently exploring this therapy for targeting CSCs.

Blocking of immune checkpoints: The immune checkpoints are cell surface molecules crucial for maintaining self-tolerance and physiological regulation of immune responses. Currently, clinical studies on antibodies to immune checkpoints in ovarian cancer are partly completed and partly in progress: for example ipilimumab (anti-CTLA-4 antibody), nivolumab (anti-PD-1 antibody), avelumab (anti-PD -L1 antibody), which are clearly effective, are still limited due to their adverse effects [86].

\section{Nanoparticle-based strategies (target agent release using CART cells or combination therapy)}

The CARs are recombinant receptors for specific antigens, which can reprogram the specificity and function of Tlymphocytes or other immune cells such as NK cells. The basic approach of the CARs is to redirect the tumor targeted T cells, called CAR-T cells (CAR-T lymphocyte), bypassing the histocompatibility complex and inducing immune reactions and resulting cytotoxicity. Car-T lymphocytes are cells of the immune system engineered in the laboratory to express a chimeric receptor, which on the outside has the structure of an antibody that specifically recognizes and hooks cancer cells, while inside it activates the signal that says to the lymphocyte to kill its prey.

Regardless of the type of T lymphocytes used, of the patient or a compatible donor or not, the idea is to manipulate the antitumor immune effector function so as to obtain what is called the "ontarget" therapeutic effect. Alongside the positive potential of T-car, there is a problem related to their use, which is assiary to the fact some targets recognized by Car-T can also be present on nontumor cells, from vascular to cardiac, from muscular to cerebral, producing the so-called "on-target off-tumor effect ": the killer that also affects out of cancer Figure 6.

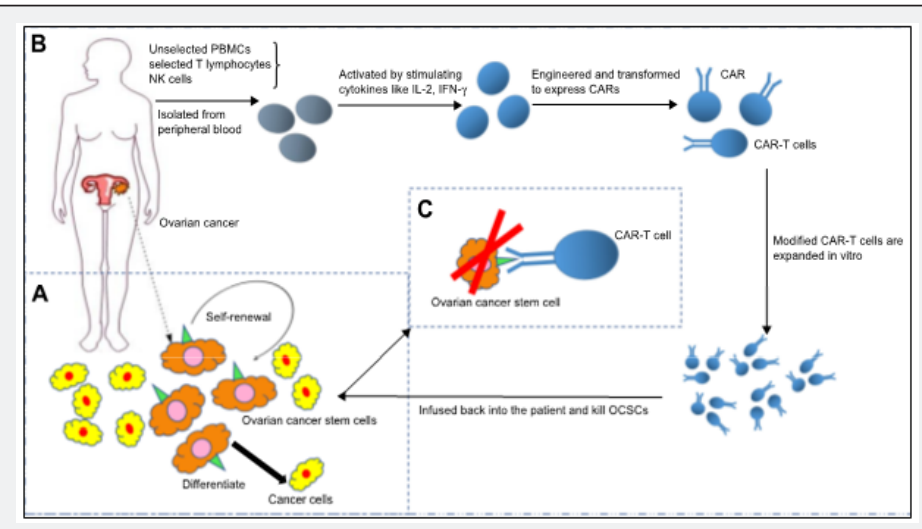

Figure 6: The CAR-T cellular immunotherapy for OCSCs.

This diagram describes how CAR-T cells affect the tumor

a) OCSCs have the ability to self-renew, asymmetrically divide and differentiate into non-tumor stem cells in the tumor architecture. The green triangle refers to the specific surface indicator on the OCSCs.

b) First, the selected T cells are isolated from the patient's peripheral blood. Second, the selected $\mathrm{T}$ cells are activated by cytokines such as IFN-?, IL-2 to enter an activated state for transduction. Third, the CAR is assembled on the $\mathrm{T}$ cell by lentiviral or other vectors. Thus, the modified $\mathrm{T}$ cells are expanded in large quantities to sacrifice size against tumor cells in vivo. Ultimately, CAR-T cells are reinfused into the patient and complete remission is expected. c) CAR recognizes the specific surface marker of OCSCs, activates adoptive cellular immune defense, collects cytokines and induces the perforin / granzyme mechanism to kill target cells. In particular, therapy with CAR-T aimed at OCSC must be combined with other therapeutic methods.

\section{Conclusion}

Given that CSCs are able to resist to the conventional therapy, and may indeed increase after chemotherapy worsening the prognosis [87], immunotherapy can make great progress in such circumstances. The "target therapy" is today the optimal method to eliminate OCSCs, since, on the one hand it captures cancer cells thanks to a specific structure and on the other it can avoid 
unforeseeable toxicity on non-cancerous cells, protecting normal tissues. Currently there is only one in vitro study that reported results of specific targeting of OCSCs throught CAR design [88], precisely because implementing a targeting of OCSCs by CAR-T appears problematic in relation to the scarcity of specific markers for their identification, as well as due to the high intratumoral heterogeneity [89]. Therefore, it is therefore understandable that the CAR-T therapy, even though it is a tried and tested treatment for many cancers, may still present unsuspected dangers, mainly due to the toxicity caused by the expression of the target antigen on non-neoplastic cells.

\section{References}

1. Bjorn B, Sae HK, Wong VW, Gurtner GC, Longaker MT, et al. (2010) Stem Cells. Plast Reconstr Surg 126(4): 1163.

2. Singec I, Jandial R, Crain A, Nikkhah G, Snyde EY, et al. (2007) The leading edge of stem cell therapeutics. Annu Rev Med 58: 313-328.

3. Mimeault M, Batra SK (2006) Concise review: Recent advances on the significance of stem cells in tissue regeneration and cancer therapies. Stem Cells 24(11): 2319-2345.

4. Beck B, Blanpain C (2013) Unravelling cancer stem cell potential. Nat Rev Cancer 13(10): 727-738.

5. Chen K, Huang YH, Chen JL (2013) Understanding and targeting cancer stem cells: therapeutic implications and challenges. Acta Pharmacol Sin 34(6): 732-740.

6. Bomken S, Fiser K, Heidenreich O, Vormoor J (2010) Understanding the cancer stem cell. Br J Cancer 103(4): 439-445.

7. Cheung TH, Rando TA (2013) Molecular regulation of stem cell quiescence. Nat Rev Mol Cell Biol 14(6): 329-340.

8. Adams JM, Strasser A (2008) Is tumor growth sustained by rare cancer stem cells or dominant clones? Cancer Res 68(11): 4018-4021.

9. Clarke MF, Dick JE, Dirks PB, Eaves CJ, Jamieson CH, et al. (2006) Cancer stem cells-perspectives on current status and future directions: AACR Workshop on cancer stem cells. Cancer Res 66(19): 9339-9344.

10. Ferrari G, Cusella-De AG, Coletta M, Paolucci E, Stornaiuolo A, et al. (1998) Muscle regeneration by bone marrow-derived myogenic progenitors. Science 279(5356): 1528-1530.

11. Quesenberry PJ, Colvin G, Dooner G, Dooner M, Aliotta JM, et al. (2007) The stem cell continuum: cell cycle, injury, and phenotype lability. Ann NY Acad Sci 1106: 20-29.

12. Lee HJ, Selesniemi K, Niikura Y, Niikura T, Klein R, et al. (2007) Bone marrow transplantation generates immature oocytes and rescues long-term fertility in a preclinical mouse model of chemotherapy-induced premature ovarian failure. J Clin Oncol 25(22): 3198-3204.

13. Bristol-Gould SK, Kreeger PK, Selkirk CG, Kilen SM, Mayo KE, et al. (2006) Fate of the initial follicle pool: empirical and mathematical evidence supporting its sufficiency for adult fertility. Developmental Biology 298(1): 149-154.

14. Virant-Klun I, Rozman P, Cvjeticanin B, Vrtacnik-Bokal E, Novakovic S, et al. (2009) Parthenogenetic embryo-like structures in the human ovarian surface epithelium cell culture in post- menopausal women with no naturally present follicles and oocytes. Stem Cells 18(1): 137150.

15. Stimpfel M, Skutella T, Cvjeticanin B, Meznaric M, Dovc P, et al. (2013) Isolation, characterization and differentiation of cells expressing pluripotent/ multipo-tent markers from adult human ovaries. Cell Tissue Res 354(2): 593-607.
16. Oatley J, Hunt PA (2012) Of mice and (wo)men: purified oogonial stem cellsfrom mouse and human ovaries. Biol Reprod 86(6): 196.

17. Silvestris E, Cafforio P, Oronzo DS, Felici C, Silvestris F, et al. (2018) In vitro differentiation of human oocyte- like cells from oogonial stem cells: single-cell isolation and molecular characterization. Hum Reprod 33(3): 464-473.

18. Wang J, Sullenger BA, Rich JN (2012) Notch signaling in cancer stem cells. Adv Exp Med Biol 727: 174-185.

19. Hambardzumyan D, Becher OJ, Rosenblum MK, Pandolfi PP, Manova-Todorova K, et al. (2008) PI3K pathway regulates survival of cancer stem cells residing in the perivascular niche following radiation in medulloblastoma in vivo. Genes Dev 22(4): 436-448.

20. Bapat SA, Mali AM, Koppikar CB, Kurrey NK (2005) Stem and progenitor-like cells contribute to the aggressive behavior of human epithelial ovarian cancer. Cancer Res 65(8): 3025-3029.

21. Thiery JP, Acloque H, Huang RY, Nieto MA (2009) Epithelial-mesenchymal transitions in development and disease. Cell 139(5): 871-890.

22. Gao Q, Geng L, Kvalheim G, Gaudernack G, Suo Z, et al. (2009) Identification of cancer stem-like side population cells in ovarian cancer cell line OVCAR-3," Ultrastructural Pathology 33(4): 175-181.

23. Zhang S, Balch C, Chan MW, Lai HC, Matei D, et al. (2008) Identification and characterization of ovarian cancer-initiating cells from primary human tumors. Cancer Res 68(11): 4311-4320.

24. Ali HR, Dawson SJ, Blows FM, Provenzano E, Pharoah PD, et al. (2011) Cancer stem cell markers in breast cancer: pathological, clinical and prognostic significance. Breast Cancer Res13(6): R118.

25. Ahmed N, Abubaker K, Findlay JK (2014) Ovarian cancer stem cells: molecular concepts and relevance as therapeutic targets. Mol Aspects Med 39: 110-125.

26. Cheong HT, Park TM, Ikeda K, Takahashi Y (2003) Cell cycle analysis of bovine cultured somatic cells by flow cytometry. Jpn J Vet Res 51(2): 95-103.

27. Xu H, Tian Y, Yuan X, Wu H, Liu Q et al. (2015) The role of CD44 in epithelial-mesenchymal transition and cancer development. Onco Targets Ther 8: 3783-3792.

28. Zoller M (2011) CD44: canacancer-initiatingcell profit from an abundantly expressed molecule? Nat Rev Cancer 11(4): 254-267.

29. Curley MD, Therrien VA, Cummings CL, Sergent PA, Koulouris CR, et al. (2009) CD133 expression defines a tumor initiating cell population in primary human ovarian cancer. Stem Cells 27(12): 2875-2883.

30. Zhang J, Guo X, Chang DY, Rosen DG, Mercado-Uribe I, et al. (2012) CD133 expression associated with poor prognosis in ovarian cancer. Mod Pathol 25(3): 456-464.

31. Ayub TH, Keyver-Paik MD, Debald M, Rostamzadeh B, Thiesler T, et al. (2015) Accumulation of ALDH1-positive cells after neoadjuvant chemotherapy predicts treatment resistance and prognosticates poor outcome in ovarian cancer. Oncotarget 6(18): 16437-16448.

32. Gao MQ Choi YP, Kang S, Youn JH, Cho NH, et al. (2010) CD24+ cells from hierarchically organized ovarian cancer are enriched in cancer stem cells. Oncogene 29(18): 2672-2680.

33. Foster R, Buckanovich RJ, Rueda BR (2013) Ovarian cancer stem cells: working towards the root of stemness. Cancer Lett 338(1): 147-157.

34. Visvader JE, Lindeman GJ (2008) Cancer stem cells in solid tumours: accumulating evidence and unresolved questions. Nat Rev Cancer 8(10): 755-768.

35. Pradhan A, Lambert QT, Reuther GW (2007) Transformation of hematopoietic cells and activation of jak2-v617f by il-27r, a component of a heterodimeric type i cytokine receptor. Proc Natl Acad Sci USA 104(47): 18502-18507. 
36. McAuliffe SM, Morgan SL, Wyant GA, Tran LT, Muto KW, et al. (2012) Targeting Notch, a key pathway for ovarian cancer stem cells, sensitizes tumors to platinum therapy. Proc Natl Acad Sci USA 109(43): E2939-E2948.

37. Silva IA, Bai S, McLean K, Yang K, Griffith K, et al. (2011) Aldehyde dehydrogenase in combina-tion with CD133 defines angiogenic ovarian cancer stem cells that portend poor patient survival. Cancer Res 71(11): 3991-4001.

38. Bhattacharya R, Kwon J, Ali B, Wang E, Patra S, et al. (2008) Role of hedgehog signaling in ovarian cancer. Clin Cancer Res 14(23): 76597666 .

39. Chen X, Horiuchi A, Kikuchi N, Osada R, Yoshida J, et al. (2007) Hedgehog signal pathway is activated in ovarian carcinomas, correlating with cell proliferation: it's inhibition leads to growth suppression and apoptosis. Cancer Sci 98(1): 68-76.

40. Bhattacharya R, Kwon J, Ali B, Wang E, Patra S, et al. (2008) Role of hedgehog signaling in ovarian cancer. Clin Cancer Res 14(23): 76597666 .

41. Fujii N, You L, Xu Z, Uematsu K, Shan J, et al. (2007) An Antagonist of Dishevelled Protein-Protein Interaction Suppresses b-Catenin-Dependent Tumor Cell Growth. Can Res 67(2): 573-579.

42. Staal FJ, Sen JM (2008) The canonical Wnt signaling pathway plays an important role in lymphopoiesis and hematopoiesis. Eur J Immunol 38(7): 1788-1794.

43. Mirzaei H, Yazdi F, Salehi R, Mirzaei HR (2016) SiRNA and epigenetic aberrations in ovarian cancer. J Cancer Res Ther 12(2): 498-508.

44. Visvader JE, Lindeman GJ (2008) Cancer stem cells in solid tumours: accumulating evidence and unresolved questions. Nat Rev Cancer 8(10): 755-768.

45. Kubitz AP, Taras EP, Boylan KL, Waldron NN, Oh S, et al. (2013) Targeting CD133 in an in vivo ovarian cancer model reduces ovarian cancer progression. Gynecol Oncol 130(3): 579-587.

46. Ferrandina G, Petrillo M, Bonanno G, Scambia G (2009) Targeting CD133 antigen in cancer. Expert Opin Ther Targets 13(7): 823-837.

47. Heider KH, Kuthan H, Stehle G, Munzert G (2004) CD44v6: a target for antibody-based cancer therapy. Cancer Immunol Immunother 53(7): 567-579.

48. Li SD, Howell SB (2010) CD44-targeted microparticles for delivery of cisplatin to peritoneal metastases. Mol Pharm 7(1): 280-290.

49. Yang X, Iyer AK, Singh A, Choy E, Hornicek FJ, et al. (2015) MDR1 siRNA loaded hyaluronic acid-based CD44 targeted nanoparticle systems circumvent paclitaxel resistance in ovarian cancer. Sci Rep 5: 8509.

50. McCann CK, Growdon WB, Kulkarni-Datar K, Curley MD, Friel AM, et al. (2011) Inhibition of Hedgehog Signaling Antagonizes Serous Ovarian Cancer Growth in a Primary Xenograft Model. PLoS One 6(11): e28077.

51. Hassan HT (2009) c-Kit expression in human normal and malignant stem cells prognostic and therapeutic implications. Leuk Res 33(1): 5-10.

52. Schilder RJ, Sill MW, Lee RB, Shaw TJ, Senterman MK, et al. (2008) Phase II Evaluation of Imatinib Mesylate in the Treatment of Recurrent or Persistent Epithelial Ovarian or Primary Peritoneal Carcinoma: A Gynecologic Oncology Group Study. J Clin Oncol 26(20): 3418-3425.

53. Matei D, Chang DD, Jeng MH (2004) Imatinib mesylate (Gleevec) inhibits ovarian cancer cell growth through a mechanism dependent on platelet-derived growth factor receptor alpha and Akt inactivation. Clinical Cancer Research 10(2): 681-690.

54. Noguera IR, Sun CC, Broaddus RR, Branham D, Levenback CF, et al (2012) Phase II trial of imatinib mesylate in patients with recur- rent platinum- and taxane-resistant low-grade serous car-cinoma of the ovary, peritoneum, or fallopian tube. Gynecol Oncol 125(3): 640-645.
55. Tse BW, Collins A, Oehler MK, Zippelius A, Heinzelmann-Schwarz VA, et al. (2014) Antibody-based immunotherapy for ovarian cancer: where are we at? Ann Oncol 25(2): 322-331.

56. Heiss MM, Murawa P, Koralewski P, Kutarska E, Kolesnik 00, et al. (2010) The trifunctional antibody catumaxomab for the treatment of malignant ascites due to epithelial cancer: Results of a prospective ran-domized phase II/III trial. Int J Cancer 127(9): 2209-222.

57. Eskander RN, Tewari KS (2013) Epithelial cell-adhesion molecule- directed trifunctional antibody immunotherapy for symp- tom management of advanced ovarian cancer. Clin Phar-macol 5(Suppl 1): 55-61.

58. Rezk Y, Yang K, Bai S, Mclean K, Johnston C, et al. (2015) Disulfiram's antineoplastic effects on ovarian cancer. J Cancer Ther 6: 1196-1205.

59. Landen CN Jr, Goodman B, Katre AA, Steg AD, Nick AM, et al. (2010) Targeting aldehyde dehydrogenase cancer stem cells in ovarian cancer Mol Cancer Ther 9(12): 3186-3199.

60. Lin J, Haffner MC, Zhang Y, Lee BH, Brennen WN, et al. (2011) Disulfiram is a DNA demethylating agent and inhibits prostate cancer cell growth. Prostate 71(4): 333-343.

61. Verma S, Stewart DJ, Maroun JA, Nair RC (1990) A randomized phase II study of cisplatin alone versus cisplatin plus disulfiram. Am J Clin Oncol 13(2): 119-124

62. Pardoll D (2003) Does the immune system see tumors as foreign or self? Annu Rev Immunol 21: 807-839.

63. Kaplan DH (1998) Demonstration of an interferon gamma-dependent tumor surveillance system in immunocompetent mice. Proc Natl Acad Sci USA 95: 7556-7561.

64. Krammer PH (2000) CD95's deadly mission in the immune system. Nature 407(6805): 789-795

65. Zitvogel L, Tesniere A, Kroemer G (2006) Cancer despite immunosurveillance: immunoselection and immunosubversion. Nat Rev Immunol 6(10): 715-727.

66. Medema JP (2001) Blockade of the granzyme B/perforin pathway through overexpression of the serine protease inhibitor PI-9/SPI-6 constitutes a mechanism for immune escape by tumors. Proc Natl Acad Sci USA 98(20): 11515-11520.

67. Terabe M (2000) NKT cell-mediated repression of tumor immunosurveillance by IL-13 and the IL-4R-STAT6 pathway. Nat Immunol 1(6): 515-520.

68. Roussel E, Gingras MC, Grimm EA, Roth JA (1995) High expression of adhesion molecules/activation markers with little interleukin-2, interferon gamma, and tumor necrosis factor beta gene activation in fresh tumor-infiltrating lymphocytes from lung adenocarcinoma. Cancer Immunol Immunother 41(1): 1-9.

69. Ding PR, Tiwari AK, Ohnuma S, Lee JW, An X, et al. (2011) The phosphodiesterase-5 inhibitor vardenafil is a potent inhibitor of ABCB1/P-glycoprotein transporter. PLoS One 6(4): e19329.

70. Li X, Li P, Zhang Y, Zhou Y, Chen X, et al. (2010) Novel Mixed Polymeric Micelles for Enhancing Delivery of Anticancer Drug and Overcoming Multidrug Resistance in Tumor Cell Lines Simultaneously. Pharm Res 27(8):1498-1511.

71. Wang Y, Cardenas H, Fang F, Condello S, Taverna P, et al. (2014) Epigenetic targeting of ovarian cancer stem cells. Cancer Res. 74(17): 49224936.

72. Chen D, Zhang Y, Wang J, Chen J, Yang C, et al. (2013) MicroRNA-200c overexpression inhibits tumorigenicity and metastasis of CD117 + CD44+ ovarian cancer stem cells by regulating epithelial-mesenchymal transition. J Ovarian Res 6(1): 50

73. Wu Q, Guo R, Lin M, Zhou B, Wang Y, et al. (2011) microRNA-200a inhibits CD133/1+ ovar- ian cancer stem cells migration and invasion by targeting E-cadherin repressor ZEB2. Gynecol Oncol 122(1): 149-154. 
74. Park YT, Jeong JY, Lee MJ, Kim KI, Kim TH, et al. (2013) microRNAs overexpressed in ovarian ALDH1-positive cells are associated with chemoresistance. J Ovarian Res 6(1): 18.

75. Cheng W, Liu T, Wan X, Gao Y, Wang H, et al. (2012) microRNA-199a targets CD44 to sup- press the tumorigenicity and multidrug resistance of ovarian cancer-initiating cells. FEBS J 279(11): 2047-2059.

76. Cowden DKD, Dahl R, Kruichak JN, Hudson LG (2009) The epidermal growth fac- tor receptor responsive miR-125a represses mesenchymal morphology in ovar- ian cancer cells. Neoplasia 11(11): 1208-1215.

77. Yeh YM, Chuang CM, Chao KC, Wang LH (2013) microRNA-138 suppresses ovar- ian cancer cell invasion and metastasis by targeting SOX 4 and HIF-1alpha. Int J Cancer 133(4): 867-878.

78. Corney DC, Hwang CI, Matoso A, Vogt M, Flesken-Nikitin A, et al. (2010) Frequent downregulation of miR-34 family in human ovarian cancers. Clin Cancer Res 16(4): 1119-1128.

79. Xu Q Liu LZ, Qian X, Chen Q Jiang Y, et al. (2012) miR-145 directly targets p70S6K1 in cancer cells to inhibit tumor growth and angiogenesis. Nucleic Acids Res 40(2): 761-774.

80. Luna JI, Grossenbacher SK, Murphy WJ, Canter RJ (2017) Targeting cancer stem cells with natural killer cell immunotherapy. Expert Opin Biol Ther 17(3): 313-324.

81. Yin T, Wang G, He S, Liu Q, Sun J, et al. (2016) Human cancer cells with stem cell-like phenotype exhibit enhanced sensitivity to the cytotox- icity of IL-2 and IL-15 activated natural killer cells. Cell Immunol 300: 41-45.

82. Silun X, Jibing C, Mingjiie Z, Zhengyi W (2017) Allogenic natural killer cell immunotherapy of sizeable ovarian cancer: A case report. Mol Clin Oncol 6(6): 903-906.

83. Krishnan V, Berek JS, Dorigo O (2017) Immunotherapy in ovarian cancer. Curr Probl Cancer 41(1): 48-63.

84. Wu D, Wang J, Cai Y (2015) Effect of targeted ovarian cancer immunotherapy using ovarian cancer stem cell vaccine. J Ovarian Res 8: 68.

85. Naujokat C (2014) Monoclonal antibodies against human cancer stem cells. Immunotherapy 6(3): 290-308.

86. Mittica G, Genta S, Aglietta M, Valabrega G (2016) Immune checkpoint inhibitors: a new opportunity in the treatment of ovarian cancer? Int J Mol Sci 17(7): pii:E1169.

87. Meacham CE, Morrison SJ (2013) Tumour heterogeneity and cancer cell plasticity. Nature 501(7467): 328-337.

88. Bonnet D, Dick JE (1997) Human acute myeloid leukemia is organized as a hierarchy that originates from a primitive hematopoietic cell. Nat Med 3(7): 730-737.

89. Karnezis AN, Cho KR, Gilks CB, Pearce CL, Huntsman DG, et al. (2017) The disparate origins of ovarian cancers: pathogenesis and prevention strategies. Nat Rev Cancer 17(1): 65-74.

\section{Your next submission with Juniper Publishers will reach you the below assets}

- Quality Editorial service

- Swift Peer Review

- Reprints availability

- E-prints Service

- Manuscript Podcast for convenient understanding

- Global attainment for your research

- Manuscript accessibility in different formats

( Pdf, E-pub, Full Text, Audio)

- Unceasing customer service

Track the below URL for one-step submission

https://juniperpublishers.com/online-submission.php 